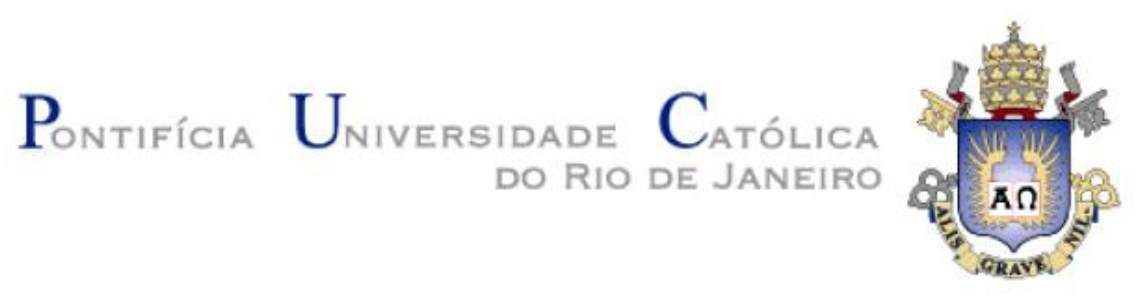

Elis Regina Lima Siqueira

\title{
Simulação de Gás Natural Injetado pelas Ventaneiras do Alto Forno
}

\section{Dissertação de Mestrado}

Dissertação apresentada ao Programa de Pósgraduação em Engenharia de Materiais e Processos Químicos e Metalúrgicos da PUC-Rio como requisito parcial para a obtenção do título de Mestre em Engenharia de Materiais e de Processos Químicos e Metalúrgicos.

Orientador: Prof. José Carlos D’Abreu

Rio de Janeiro

Agosto de 2014 


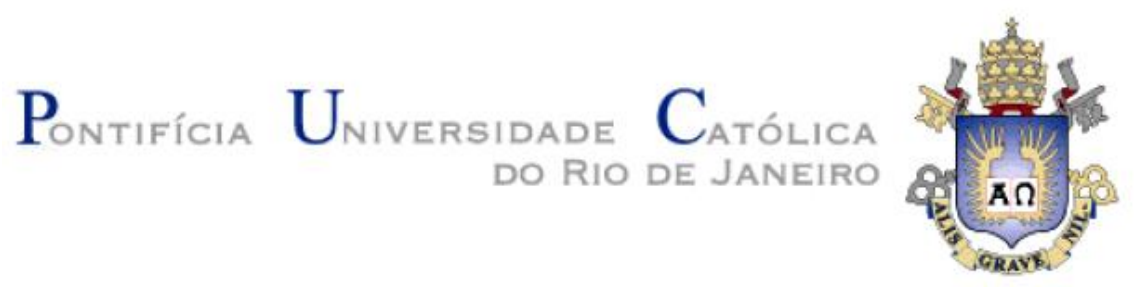

\section{Elis Regina Lima Siqueira}

\section{Simulação de Gás Natural Injetado pelas Ventaneiras do Alto Forno}

Dissertação apresentada como requisito parcial para obtenção do grau de Mestre pelo Programa de Pósgraduação em Engenharia de Materiais e de Processos Químicos e Metalúrgicos da PUC-Rio. Aprovada pela Comissão Examinadora abaixo assinada.

Prof. José Carlos D’Abreu Orientador Departamento de Engenharia de Materiais - PUC-Rio

Prof. Helio Marques Kohler Consultor TECHN-NOS

Prof. Francisco José Moura Departamento de Engenharia de Materiais - PUC-Rio

Prof. José Eugênio Leal Coordenador Setorial do Centro Técnico Científico (PUC-Rio)

Rio de Janeiro, 30 de agosto de 2014 
Todos os direitos reservados. É proibida a reprodução total ou parcial do trabalho sem autorização da universidade, do autor e do orientador.

\section{Elis Regina Lima Siqueira}

Graduou-se em Engenharia de Materiais (Universidade Federal do Pará - Brasil, 2011), Trabalhou e obteve experiência profissional na indústria siderúrgica SINOBRÁS.

Ficha Catalográfica

Siqueira, Elis Regina Lima

Simulação de gás natural injetado pelas ventaneiras de alto forno / Elis Regina Lima Siqueira ; orientador: José Carlos D'Abreu. 2014.

$90 \mathrm{f.} ; 30 \mathrm{~cm}$

Dissertação (mestrado)-Pontifícia Universidade Católica do Rio de Janeiro, Departamento de Engenharia de Materiais, 2014.

Inclui bibliografia

1. Engenharia de materiais - Teses. 2. Alto forno. 3. Ventaneiras. 4. Injeção de gás natural. 5. Simulação computacional. I. D'Abreu, José Carlos. II. Pontifícia Universidade Católica do Rio de Janeiro. Departamento de Engenharia de Materiais. III. Título.

CDD: 620.11 
O único homem que está isento de erros, é aquele que não arrisca acertar Albert Einstein 


\section{Agradecimentos}

Agradeço a minha família pelo apoio, dedicação e incentivo ao desenvolvimento desse projeto. De uma forma sutil eles foram a força motriz durante os anos dedicados ao desenvolvimento deste.

Ao CNPq e à PUC-Rio, pelos auxílios concedidos, sem os quais este trabalho não poderia ter sido realizado.

Aos meus professores do DEMA- Departamento de Engenharia de Materiais da PUCRio em especial ao professor Francisco Moura.

Ao meu orientador José Carlos D'Abreu, que sempre me colocou diante de desafios, permitindo dessa forma o meu desenvolvimento acadêmico. A sua experiência proveniente de anos dedicados ao setor siderúrgico refletiu nas aulas esclarecedoras e dinâmicas referentes a processos siderúrgicos, agradeço pela contribuição!

Ao professor Hélio Kohler, por estar sempre disposto em auxiliar, dando sempre o melhor de si.

Ao Jorge Luiz, pela gentileza em colaborar para o desenvolvimento deste, através de dados, apostilas e esclarecimentos referentes à injeção de gás natural em altos fornos siderúrgico. Todas essas informações foram de fundamental importância, no sentido de dar mais credibilidade ao projeto, pois, são relacionadas a resultados reais do processo de produção de ferro gusa em um alto forno siderúrgico.

Ao Thiago Konopka, por ter concedido o programa de simulação da combustão, o mecanismo cinético e por ter sido sempre solicito, com relação às minhas dúvidas referentes ao programa.

Aos membros do grupo de siderurgia: Raimundo Nonato, Enrique Dionísio, Jerson Alvarado e Efrain. Ao grande Ronald Mejia Sanches, um amigo sempre disposto a contribuir. Muito obrigada! 


\section{Resumo}

Siqueira, Elis Regina Lima; D’Abreu, José Carlos. Simulação de Gás Natural Injetado pelas Ventaneiras do Alto Forno. Rio de Janeiro, 2014. 90p. Dissertação de Mestrado - Departamento de Engenharia de Materiais, Pontifícia Universidade Católica do Rio de Janeiro.

O alto forno é um reator metalúrgico cujo objetivo consiste na produção de ferro-gusa. O consumo de combustível/redutor no processo de redução de minério de ferro em altos fornos, representa mais de $50 \%$ do custo do gusa. No sentido de aumentar a produtividade e reduzir o consumo de combustível/redutor são empregadas técnicas de injeção de combustíveis auxiliares pelas ventaneiras dos altos fornos. A combustão de gás natural $(\mathrm{GN})$ injetado nas ventaneiras produz grande quantidade de hidrogênio, esse gás é melhor redutor se comparado ao monóxido de carbono, pois ele possui velocidade de reação maior com os óxidos de ferro e, além disso, a geração de $\mathrm{CO}_{2}$ no processo de redução é diminuída quando comparado ao uso do carvão pulverizado (PCI), que é atualmente o material de injeção mais usado no Brasil. Este trabalho propõe a simulação da combustão de GN injetado pelas ventaneiras de um alto forno, utilizando o software CHEMKIN. As simulações provenientes deste software são amplamente utilizadas para otimização da combustão, sendo possível explorar rapidamente o impacto das variáveis de projeto sobre o desempenho do processo. Os resultados provenientes dessa simulação computacional em condições típicas de alto forno permitiram a previsão da temperatura de chama adiabática e a quantificação dos gases redutores de óxidos de

ferro: $\mathrm{H}_{2}$ e CO. A partir da variação dos parâmetros de processo foi possível obter resultados úteis para a tomada de decisão, visando controlar e otimizar o processo.

\section{Palavras-chave}

Alto-forno; ventaneiras; injeção de gás natural; simulação computacional. 


\section{Abstract}

Siqueira, Elis Regina Lima; D'Abreu, José Carlos (advisor). Natural Gas Simulation Injected for tuyeres of blast furnaces Steel. Rio de Janeiro, 2014. 90p. MSc. Dissertation - Departamento de Engenharia de Materiais, Pontifícia Universidade Católica do Rio de Janeiro.

The blast furnace is a metallurgical reactor whose goal is to produce pig iron. The fuel / reductant in the reduction of iron ore in the blast furnace process, represents more than $50 \%$ of the cost of the iron. In order to increase the productivity of the blast furnace and reduce fuel consumption / reducer injection techniques are employed by tuyeres of materials that act as fuel / reducer. The combustion of natural gas injected into the tuyeres produces large amounts of hydrogen, which replaces part of the carbon monoxide as reducing gas in the tank. The hydrogen gas is better compared to the reductant carbon monoxide, because it has reaction rate with the iron oxides and, moreover, the $\mathrm{CO} 2$ generation in the process of reduction is decreased when compared to the use of pulverized coal (PCI), which is currently the material most commonly used injection by tuyeres in Brazil. This paper proposes the simulation of combustion of natural gas injected into the tuyeres of a blast furnace, using the CHEMKIN software package. Simulations from this software are widely used for optimization of combustion, which can quickly explore the impact of design variables on the performance of the process, using accurate models of chemical kinetics. The computer simulation results from the combustion of natural gas at typical conditions of blast furnaces allowed the prediction of the adiabatic flame temperature and the reaching of the reducing gases of iron oxides: $\mathrm{H} 2$ and $\mathrm{CO}$. From the variation of process parameters was possible to obtain useful results in order to control and optimize the process.

\section{Keywords}

Blast furnace; tuyeres; injection of natural gas; computer simulation. 


\section{Sumário}

1. Introdução 15

$\begin{array}{ll}\text { 1.1.Estrutura do texto } & 18\end{array}$

2. Objetivos 20

2.1. Objetivos Específicos $\quad 20$

3. Revisão bibliográfica 21

3.1. Produção de ferro-gusa em altos fornos 21

3.2. Divisão do Alto Forno em regiões 23

3.3. Conjunto de sopro do ar de combustão 25

3.4. Zona de combustão (raceway) 28

3.5. Combustíveis/redutores empregados no alto forno 28

3.5.1. Carvão mineral coqueificável 28

3.5.2. Carvão vegetal 29

3.6. Função dos combustíveis/redutores no alto forno 29

3.6.1. Combustível 30

3.6.2. Redutor de óxidos de ferro 31

3.6.2.1. Diagrama de Chaudron 32

3.6.3. Permeabilizador e agente estrutural da carga 33

3.6.4. Fornecedor de carbono 33

3.7. Injeção de combustíveis auxiliares 33

3.7.1. Injeção de carvão pulverizado 34

3.7.2. Injeção de óleo combustível 35

3.7.3. Injeção de plástico 36

3.7.4. Injeção de borracha de pneu 37

3.7.5. Injeção de pó de balão 38

3.7.6. Injeção de gás natural 38 
3.8. Redução de óxidos de ferro por hidrogênio gasoso 39

3.8.1. Velocidade de redução de óxidos de ferro 40

3.9. Sistema de equilíbrio $\mathrm{Fe}-\mathrm{O}-\mathrm{C}$ e $\mathrm{Fe}-\mathrm{O}-\mathrm{H}$

3.10. Redução dos óxidos de ferro em misturas de $\mathrm{CO} \mathrm{e} \mathrm{H}_{2}$

3.11. Oxidação de hidrocarbonetos $\quad 44$

3.11.1. Mecanismo do gás natural 44

3.12. Relação ar/combustível 45

4. Metodologia $\quad 47$

4.1. Software aplicado para simulação da combustão 48

4.2. Mecanismo de reação aplicado no software CHEMKIN 48

4.3. Reator Perfeitamente Agitado 49

4.4. Variáveis de projeto $\quad 50$

5. Resultados e discussões 54

5.1. Influência do enriquecimento de $\mathrm{O} 2$ do ar de combustão na temperatura de chama adiabática 54

5.2. Efeito do aquecimento do ar de combustão na temperatura de chama adiabática $\quad 60$

5.3. Efeito da vazão de GN na temperatura de chama adiabática 63

5.4. Efeito da formação de $\mathrm{CO}_{2}$ na temperatura de chama adiabática 64

5.5. Efeito da riqueza da mistura na produção de $\mathrm{CO}, \mathrm{H}_{2}, \mathrm{CO}_{2}$ e $\mathrm{H}_{2} \mathrm{O} \quad 67$

5.6. Efeito da riqueza da mistura no consumo de $\mathrm{CH}_{4}$ e $\mathrm{O}_{2}$

5.7. Estudo paramétrico a partir da vazão de Gás Natural 76

5.8. Efeito da injeção de GN na produção de ferro gusa líquido 82

5.9. Taxa de substituição ( $\mathrm{Kg}$ coque/ $\mathrm{Nm}^{3}$ de Gás Natural) 84

6. Conclusões $\quad 85$

REFERÊNCIAS BIBLIOGRÁFICAS $\quad 88$ 


\section{Lista de figuras}

Figura 1 - Diagrama da oxidação do $\mathrm{CH} 4$, pressão atmosférica (Kee, 2013 ).

Figura 2 - Representação simplificada do processo de produção de ferro gusa no alto- forno (RIZZO, 2009)

Figura 3 - Regiões de um alto-forno de acordo com o seu perfil (RIZZO, 2009)

Figura 4 - Exemplo do interior de um alto-forno com destaque para o cadinho e as ventaneiras (Kobe Steel)

Figura 5 - (a) Arranjo das ventaneiras no interior do forno;

(b) Detalhes internos da ventaneira (MCNALLY et al, 2011)

Figura 6 - Representação esquemática do conjunto de sopro de ar de combustão (RIZZO,2009)

Figura 7 - Sistema externo do sopro de ar com destaque para o anel de vento (RIZZO, 2009)

Figura 8 - Diagrama de equilíbrio da reação de Boudouard, pressão 1 atm (QUINTANILLA, 2012)

Figura 9 - Diagrama de Chaudron: Diagrama de estabilidade do sistema Fe-O-C em função da temperatura

Figura 10 - Influência do percentual de gás redutor $(\mathrm{CO}$ ou H2) em misturas $(\mathrm{CO}+\mathrm{N} 2)$ e $(\mathrm{H} 2+\mathrm{N} 2)$ sobre a cinética de redução, (HANSEN, 2000)

Figura 11 - Diagrama de equilibrio Fe-O-C e Fe-O-H adicionado a reação de Boudouard

Figura 12 - Digrama de oxi-redução para o sistema Fe-O-C-H

Figura 13 - Representação esquemática do reator

perfeitamente misturado (Fogler, 2009) 
Figura 14: Influência do enriquecimento de $\mathrm{O} 2$ do ar de combustão na temperatura de chama adiabática. Vazão de GN: 2,00Nm2/seg. Vazão do ar de combustão: 72,45 Nm3/seg. Temperatura de aquecimento do ar: $1020^{\circ} \mathrm{C}$.

Figura 15 - Efeito do enriquecimento de oxigênio no ar de combustão.Ar pré-aquecido a $1020^{\circ} \mathrm{C}$. Vazão de GN constante: $0,18 \mathrm{Nm} 3 / \mathrm{seg}$.

Figura 16 - Efeito do enriquecimento de oxigênio no ar de combustão.Ar pré-aquecido a $1020^{\circ} \mathrm{C}$. Vazão de GN constante: $2,00 \mathrm{Nm} 3 / \mathrm{seg}$.

Figura 17 - Efeito do enriquecimento de oxigênio no ar de combustão.Ar pré-aquecido a $1020^{\circ} \mathrm{C}$. Vazão de GN constante: $3,92 \mathrm{Nm} 3 / \mathrm{seg}$.

Figura 18 - Efeito da temperatura de aquecimento do ar na temperatura de chama adiabática. Ar pré aquecido a $1020^{\circ} \mathrm{C}$ e $1350^{\circ} \mathrm{C}$, com $3,21 \%$ de enriquecimento de O2. Vazão de GN constante: $0,18 \mathrm{Nm} 3 / \mathrm{seg}$

Figura 19 - Efeito da temperatura de aquecimento do ar na temperatura de chama adiabática. Ar pré aquecido a $1020^{\circ} \mathrm{C}$ e $1350^{\circ} \mathrm{C}$, com $3,21 \%$ de enriquecimento de O2. Vazão de GN constante: $2,00 \mathrm{Nm} 3 / \mathrm{seg}$

Figura 20 - Efeito da temperatura de aquecimento do ar na temperatura de chama adiabática. Ar pré aquecido a $1020^{\circ} \mathrm{C}$ e $1350^{\circ} \mathrm{C}$, com $3,21 \%$ de enriquecimento de O2. Vazão de GN constante: $3,92 \mathrm{Nm} 3 / \mathrm{seg}$

Figura 21 - Efeito da vazão de GN na temperatura de chama adiabática. Vazão do ar: 72,45 Nm3/seg. temperatura de aquecimento do ar: $1020^{\circ} \mathrm{C}$. Enriquecimento de O2: $0,00 \%$

Figura 22 - Influência da formação de CO2 na temperatura de chama adiabática. Ar de combustão pré aquecido a 
$1020^{\circ} \mathrm{C}$ e enriquecido com $3,21 \%$ O2, vazão entre 28,92

- 106,55 Nm3/seg com vazão de GN: 0,18Nm3/seg

Figura 23 - Influência da formação de CO2 na temperatura

de chama adiabática. Ar de combustão pré aquecido a $1020^{\circ} \mathrm{C}$ e enriquecido com $3,21 \%$ O2, vazão entre 28,92

- 106,55 Nm3/seg com vazão de GN: 2,00Nm3/seg

Figura 24 - Influência da formação de $\mathrm{CO} 2$ na temperatura de chama adiabática. Ar de combustão pré aquecido a $1020^{\circ} \mathrm{C}$ e enriquecido com $3,21 \%$ O2, vazão entre 28,92 - 106,55 $\mathrm{Nm} 3 /$ seg com vazão de GN: 3,92Nm3/seg

Figura 25 - Produção de H2 e CO a partir da combustão de GN com ar de combustão pré aquecido a $1020^{\circ} \mathrm{C}$ e enriquecido com $3,21 \%$ de oxigênio. Vazão de GN constante: $0,18 \mathrm{Nm} 3 / \mathrm{seg}$, vazão do sopro de ar variando entre: 28,92 - 106,55 Nm3/seg Figura 26 - Produção de H2 e CO a partir da combustão de GN com ar de combustão pré aquecido a $1020^{\circ} \mathrm{C}$ e enriquecido com $3,21 \%$ de oxigênio. Vazão de GN constante: $2,00 \mathrm{Nm} 3 / \mathrm{seg}$, vazão do soprode ar variando entre: 28,92 - 106,55 Nm3/seg Figura 27 - Produção de H2 e CO a partir da combustão de GN com ar de combustão pré aquecido a $1020^{\circ} \mathrm{C}$ e enriquecido com $3,21 \%$ de oxigênio. Vazão de GN constante: 3,92Nm3/seg, vazão do sopro de ar variando entre: 28,92 - 106,55 Nm3/seg Figura 28 - Produção de $\mathrm{H} 2 \mathrm{O}$ e $\mathrm{CO}$ a partir da combustão de GN com ar de combustão pré aquecido a $1020^{\circ} \mathrm{C}$ e enriquecido com $3,21 \%$ de oxigênio. Vazão de GN constante: $0,18 \mathrm{Nm} 3 / \mathrm{seg}$, vazão do sopro de ar variando entre: 28,92 - 106,55 Nm3/seg Figura 29 - Produção de $\mathrm{H} 2 \mathrm{O}$ e $\mathrm{CO}$ a partir da combustão de GN com ar de combustão pré aquecido a $1020^{\circ} \mathrm{C}$ e enriquecido com 3,21\% de oxigênio. Vazão de GN constante: 2,00 Nm3/seg, vazão do sopro de ar variando entre: 28,92 - 106,55 Nm3/seg 72 Figura 30 - Produção de $\mathrm{H} 2 \mathrm{O}$ e $\mathrm{CO}$ a partir da combustão de GN 
com ar de combustão pré aquecido a $1020^{\circ} \mathrm{C}$ e enriquecido com $3,21 \%$ de oxigênio. Vazão de GN constante: 3,92 Nm3/seg, vazão do sopro de ar variando entre: 28,92 - 106,55 Nm3/seg

Figura 31 - Disponibilidade de $\mathrm{O} 2$ e $\mathrm{CH} 4$ a partir da combustão de GN com vazão de $0,18 \mathrm{Nm} 3 / \mathrm{seg}$ e ar de combustão enriquecido com $3,21 \% \mathrm{O} 2$, pré aquecido a $1020^{\circ} \mathrm{C}$, vazão entre 28,92 $-106,55 \mathrm{Nm} 3 / \mathrm{seg}$

Figura 32 - Disponibilidade de $\mathrm{O} 2$ e CH4 a partir da combustão de GN com vazão de 2,00 Nm3/seg e ar de combustão enriquecido com $3,21 \%$ O2, pré aquecido a $1020^{\circ} \mathrm{C}$ e com vazão entre $28,92-106,55 \mathrm{Nm} 3 / \mathrm{seg}$ Figura 33 - - Disponibilidade de $\mathrm{O} 2$ e CH4 a partir da combustão de GN com vazão de 3,92 Nm3/seg e ar de combustão enriquecido com $3,21 \%$ O2, pré aquecido a $1020^{\circ} \mathrm{C}$ e com vazão entre 28, $92-106,55 \mathrm{Nm} 3 / \mathrm{seg}$

Figura 34 - Temperatura de chama adiabática proveniente da combustão de GN com ar de combustão pré aquecido a $1020^{\circ} \mathrm{C}$. Figura 35 - Produção de CO2 a apartir da combustão de GN com ar pré aquecido: $1020^{\circ} \mathrm{C}$. vazão: 28,92, 62,44 e 106,55 Nm3/seg. Figura 36 - Produção de $\mathrm{H} 2 \mathrm{O}$ a apartir da combustão de GN com ar pré aquecido: $1020^{\circ} \mathrm{C}$. vazão: $28,92,62,44$ e $106,55 \mathrm{Nm} 3 / \mathrm{seg}$.

Figura 37 - Produção de $\mathrm{CO}$ a apartir da combustão de GN com ar pré aquecido: $1020^{\circ} \mathrm{C}$. Vazão: 28,92, 62,44 e 106,55 Nm3/seg. 80 Figura 38 - Produção de H2 a partir da combustão de GN com ar pré aquecido: $1020^{\circ} \mathrm{C}$. Vazão: 28,92, 62,44 e 106,55 Nm3/seg. 81 Figura 39 - Disponibilidade de $\mathrm{CH} 4$ a partir da combustão de GN com ar pré aquecido: $1020^{\circ} \mathrm{C}$. Vazão: 28,92, 62,44 e 106,55 Nm3/seg. 82 Figura 40 - Produção mensal de ferro gusa a partir de distintas taxas de consumo de coque e de gás natural 


\section{Lista de tabelas}

Tabela 1 - Relação H/C de algumas tecnologias de redução 44

Tabela 2 - Composição do gás natural 51

Tabela 3 - Composição do ar de combustão 52

Tabela 4 - Vazão volumétrica do comburente e do combustível 52

Tabela 5 - Riqueza da mistura para distintos \% de enriquecimento de $\mathrm{O} 2$

Tabela 6 - Riqueza da mistura para distintos \% de enriquecimento de $\mathrm{O} 2 \quad 58$

Tabela 7 - Riqueza da mistura para distintos \% de enriquecimento de $\mathrm{O} 2$

Tabela 8 - Taxa de substituição Kg coque/Nm3 de GN 84 